\title{
2010 CSHP National Awards Program Winners Programme national des prix 2010 de la SCPH : lauréats et lauréates
}

The winner of the Distinguished Service Award (sponsored by Ortho Biotech Division of JanssenOrtho Inc.) is Emily Musing (University Health Network, Toronto, ON).

The winner of the Isabel E. Stauffer Meritorious Service Award (sponsored by Pharmaceutical Partners of Canada Inc.) is Victoria Sills (St. Mary's General Hospital, Kitchener, ON).
The winners of the New Hospital Pharmacy Practitioner Award (sponsored by Sandoz Canada Inc.) are Rochelle M Gellatly (St. Paul's Hospital, Vancouver, BC) and Erin Cashin (Alberta Health Services, Red Deer, AB).

The winner of the Hospital Pharmacy Student Award (sponsored by the Canadian Society of Hospital Pharmacists [CSHP] and the Canadian Association of Pharmacy Students and Interns [CAPSI]) is Christine Leong (Winnipeg, MB).
Management and Leadership Best Practices Award Sponsored by Apotex Inc.

1. Obtaining the Best Possible Medication History: Comparison of Pharmacy Technician versus Pharmacist Obtained Medication Histories in the Emergency Department (completed at The Moncton Hospital, Moncton, NB)

Rochelle Johnston and Lauza Saulnier

Sponsored by Hospira Healthcare Corporation

2. Development of a Supervisory Skills Course for Hospital Pharmacy Workplaces (completed at the Winnipeg Regional Health Authority, Winnipeg, MB)

Donna M.M. Woloschuk and Colette B. Raymond

Patient Care Enhancement Award

Sponsored by AstraZeneca Canada Inc.

1. Administration of $24 \%$ Sucrose for Procedural Pain Management in Neonates: An Evidence-Based Approach to Changing Practice (completed at the Children's Hospital of Eastern Ontario, Ottawa, ON)

Régis Vaillancourt

Sponsored by TEVA Novopharm

2. Pharmacist and Physician Collaborative Prescribing for Medication Renewals within a Primary Health Centre (completed at West Winds Primary Health Centre, Saskatoon, SK)

Angela McKinnon and Derek Jorgenson

Pharmacotherapy Best Practices Award Sponsored by Merck Frosst Canada Ltd.

1. Once Daily Gentamicin Dosing in Febrile Neutropenic Children (completed at The Hospital for Sick Children, Toronto, ON)

Miriam Inparajah, L. Lee Dupuis, and Sabrina Boodhan

Sponsored by Pfizer Canada

2. A Practical Method of Providing Pharmaceutical Care in the Intensive Care Unit: FASTHUG-MAIDENS (completed at Royal Columbian Hospital, Burnaby, BC)

Vincent H. Mabasa, Douglas L. Malyuk, Elisa-Marie Weatherby, and Alice Chan

\section{Safe Medication Practices Award}

Sponsored by Baxter Corporation

1. Attitudes and Behaviors of Pharmacy Staff toward Near Misses: Reliability and Validity of a Survey Tool (completed at Winnipeg Regional Health Authority, Winnipeg, MB)

Sarah Miller, Colette Raymond, Hannah Wheaton, and Nick Honcharik

Sponsored by Hospira Healthcare Corporation

2. Medication Reconciliation and the Role of the Pharmacy Technician: A Pilot Project with Acute Care Cardiac Services (completed at New Brunswick Heart Centre, Saint John, NB)

Nadia Chishti, Trudy Arbo, Moira Wilson, and Jaclyn LeBlanc

Specialties in Pharmacy Practice Award

Sponsored by Bristol-Myers Squibb Canada

1. Evaluation of Creatinine-Based Formulas in Dosing Adjustment of Cancer Drugs Other Than Carboplatin (completed at BC Cancer Agency, Vancouver, BC)

Sarah Jennings and Mário L. de Lemos

Sponsored by Hospira Healthcare Corporation

2. Evaluation of Parenteral Vitamin B12 Administration in Hemodialysis Patients (completed at Vancouver General Hospital, Vancouver, BC)

Victoria Su and Karen Shalansky

Teaching, Learning, and Education Award Sponsored by Eli Lilly Canada

1. The Development and Evaluation of a Student Pharmacist Clinical Teaching Unit Utilizing Peer Assisted Learning (completed at Red Deer Regional Hospital, Red Deer, AB) Adrienne Lindblad, Jason Howorko, Richard Cashin, Carmen Leung and Erin Cashin

The award-winning abstracts are published exactly as submitted by the authors and have not undergone any copyediting by the Canadian Journal of Hospital Pharmacy.

Le Journal canadien de la pharmacie hospitalière n'a pas soumis les résumés gagnant des prix à une révision linguistique et les publie ici tels que remis par les auteurs. 


\section{Obtaining the Best Possible Medication History: Comparison of Pharmacy Technician versus Pharmacist Obtained Medication Histories in the Emergency Department}

\section{Management and Leadership Best Practices Award, sponsored by Apotex Inc.}

Rochelle Johnston ${ }^{1}$, Lauza Saulnier', Odette Gould ${ }^{1,2}$

${ }^{1}$ Horizon Health Network, Zone 1 Moncton, ${ }^{2}$ Mount Allison University

Background: Obtaining an accurate and complete medication list (e.g. best possible medication history (BPMH)) is the first step in completing medication reconciliation. The ability of pharmacy technicians to obtain medication histories compared to pharmacists has not been formally assessed.

Objective: Study objectives were to assess whether pharmacy technicians can perform a BPMH as accurately and completely as pharmacists, and determine if both groups met national norms for unintentional discrepancies and success index for medication reconciliation. Methods: Patients presenting to the emergency department were prospectively enrolled to be interviewed separately by a pharmacist and a technician, with information recorded on a standard medication reconciliation form. Forms were compared following each set of interviews, and discrepancies were clarified with the patient. Pharmacy technicians were trained in taking BPMH prior to patient enrolment.

Results: Fifty-nine patients were included. Pharmacists and technicians did not differ significantly in whether their patients had prescription $\left(\mathrm{X}^{2}(1, \mathrm{~N}=59)=1.11, \mathrm{p}=.29\right)$ or OTC $\left(\mathrm{X}^{2}(1, \mathrm{~N}=59)=.15, \mathrm{p}=.70\right)$ discrepancies. Mean prescription and OTC discrepancies per patient were not significantly different between the two groups $(\mathrm{t}(58)=.15, \mathrm{p}=$ $.88 ; \mathrm{t}(58)=-.22, \mathrm{p}=.83)$. Both groups were significantly lower than the national average for unintentional discrepancies per patient and significantly higher than the national average for success index.

Conclusions: Trained pharmacy technicians can obtain a BPMH with as much accuracy and completeness as pharmacists. Both groups were significantly superior to the national average for unintentional discrepancies and success index for medication reconciliation.

Key Words: medication reconciliation, med-rec, pharmacy technician, best possible medication history, BPMH, emergency department

\section{Development of a Supervisory Skills Course for Hospital Pharmacy Workplaces}

Management and Leadership Best Practices Award, sponsored by Hospira Healthcare Corporation

Donna M.M. Woloschuk, Colette B. Raymond

Winnipeg Regional Health Authority Pharmacy Program, Winnipeg, Manitoba

Introduction: Many Canadian hospital pharmacies are experiencing difficulties recruiting supervisory personnel. We developed and implemented a "learning by doing" course to enhance acquisition and application of supervisory knowledge and skill.

Description of Concept: Course goals were to provide learners with experience in supervisory roles, and to enhance the application of supervisory knowledge and skill. Learners were expected to apply skills in the supervision of pharmacy operations and human resources, and perform supervisory documentation. Course design emphasized a constructivist framework incorporating authentic learning and reflective seminars with workplace self-directed learning. Preceptors assisted learners to achieve course goals.

Results and Evaluation: Eighteen pharmacy technicians were admitted to the program; one withdrew due to a job change. All learn- ers successfully completed the course. Two months after the course, learners and preceptors described enhanced learner organization, time management, leadership, communication, and conflict resolution skills, and increased confidence, maturity, and ability to supervise staff. Learner evaluations revealed a broadened perspective of pharmacy. Preceptors valued learners' enhanced skills and increased enthusiasm. Six participants have secured supervisory positions to date.

Relevance to Practice: A supervisory skills course grounded in constructivist theory resulted in high learner satisfaction with learning outcomes.

Key words: supervisory skills, pharmacy technicians, workplace professional development

\section{Administration of $\mathbf{2 4 \%}$ Sucrose for Procedural Pain Management in Neonates: An Evidence-Based Approach to Changing Practice \\ Patient Care Enhancement Award, sponsored by AstraZeneca Canada Inc.}

Régis Vaillancourt, Elena Pascuet, Mary MacNeil, Karen McCoy, Brett Mulholland, Vassant Dabee

Children's Hospital of Eastern Ontario (CHEO), Ottawa, ON

Background: Use of $24 \%$ sucrose has been demonstrated as a simple, safe and effective pain-relieving intervention for neonatal pain management. The study objective was to monitor the implementation and utilization of $24 \%$ sucrose by nurses for procedural pain for all neonates admitted to the Infant/Toddler inpatient unit.

Methods: Key stakeholders formed part of the multidisciplinary planning team and implemented changes using a rapid cycle PDSA improvement process. Performance measures were tracked by retrospective chart auditing of all medically stable inpatients up to 1month of corrected age.

Results: A total $85 \%$ of patients $(n=41)$ had sucrose initiated on the physician order sheet by the nurse and sucrose given prior to $67 \%$ $(\mathrm{n}=98)$ of medical procedures. The rate of sucrose initiation on the physician order sheet was never found to be below $75 \%$. Sucrose was used most often prior to blood work $(\mathrm{n}=39)$, and less frequently prior to suctioning $(\mathrm{n}=59)$.

Conclusions: Active involvement of unit champions, support and collaboration from unit leadership, pharmacy and staff physicians were strong facilitators for staff to adopt $24 \%$ sucrose for procedural pain. Since hospital-wide implementation, usage of wardstock sucrose units has increased 6 fold.

Key words: Analgesia; Neonate; Pain; Patient Care; Policy Implementation; Sucrose

\section{Pharmacist and Physician Collaborative Prescribing for Medication Renewals within a Primary Health Centre}

\section{Patient Care Enhancement Award, sponsored by TEVA Novopharm}

Angela McKinnon, Saskatoon Health Region

Derek Jorgenson, University of Saskatchewan

Background: Improving the process utilized to reorder chronic prescriptions in family physician practices may lead to safer and more effective medication management in the primary health care system. We sought to determine if there was an improvement in medication management when a pharmacist collaborated with family physicians to prescribe medication renewals requested by fax. 
Methods: Prospective, non-randomized controlled trial. Medication renewal requests were forwarded to the pharmacist on days when he was working (Intervention Group). The pharmacist made an assessment for drug therapy issues that might preclude safe and effective prescribing of the medication. The pharmacist and physician then made a collaborative decision to authorize the requested medication or to request additional intervention(s) first (i.e., perform lab test). When the pharmacist was not working, the physicians managed the renewal requests independently (Control Group). Primary outcomes were: refill requests authorized with no recommendations, drug-related problems found, new monitoring ordered, and new appointments scheduled with a health provider.

Results: 181 refill requests were included (94 in control group and 87 in intervention group). The control group had significantly more requests authorized with no recommendations $(75.5 \%$ vs. $52.9 \%$, $\mathrm{p}<0.001)$. The intervention group had significantly more: drug related problems identified ( 26 vs. $10, \mathrm{p}<0.031$ ); medication changes made (24 vs. $10, \mathrm{p}<0.044$ ); and new appointments scheduled with the family physician (31 vs. $21, \mathrm{p}<0.049$ ).

Conclusion: There is an improvement in medication management when a pharmacist collaborates with family physicians to prescribe medication renewals. The collaborative model created significantly more activity with each renewal request, which reflects an improvement in the process of care.

Key Words: Pharmacist, prescription refills, collaboration, primary health care

\section{Once Daily Gentamicin Dosing in Febrile Neutropenic Children}

\section{Pharmacotherapy Best Practices Award, sponsored by Merck Frosst Canada Ltd.}

Miriam Inparajah (The Scarborough Hospital-General Division, Toronto, ON), Cecile Wong, Cathryn Sibbald, Sabrina Boodhan, Eshetu G. Antenafu, Ahmed Naqvi, L. Lee Dupuis (The Hospital for Sick Children, Toronto, ON)

Background: Our once daily gentamicin (ODG) dosing guideline was developed in children undergoing bone marrow transplant.

Objectives: To evaluate an existing ODG dosing guideline in children with febrile neutropenia resulting from conventional chemotherapy (FN) and to develop a new more reliable dosing guideline.

Methods: Retrospective review of children with FN who received ODG. Pharmacokinetic parameters were calculated according to standard first-order, one-compartment equations; initial ideal doses that achieved a concentration at the end of the infusion (Cmax) of 23 $\mathrm{mg} / \mathrm{L}$ were calculated. Univariate analysis or student's t test were used to identify significant relationships between pharmacokinetic parameters, patient age and sex. The recursive binary partitioning method was used to determine critical values of age. ANOVA was used to compare the different levels obtained after recursive partitioning technique. Ideal ODG dosing guidelines were developed using the mean dose required to achieve the target Cmax $(23 \mathrm{mg} / \mathrm{L})$.

Results: 111 patients were included. The existing ODG guideline achieved a Cmax within the target range in 20 patients (18\%). A new initial ODG guideline was developed: 1 to < 6 years: $10.5 \mathrm{mg} / \mathrm{kg} / \mathrm{dose}$; girls $\geq 6$ years: $9.5 \mathrm{mg} / \mathrm{kg} /$ dose; and boys $\geq 6$ years: $7.5 \mathrm{mg} / \mathrm{kg} / \mathrm{dose}$. Conclusion: The proposed initial ODG guideline for febrile neutropenic children is predicted to achieve a Cmax within or above the target range in $73 \%$ of cases.

Key words: once daily gentamicin, aminoglycosides, pharmacokinetics, pediatrics, neutropenia, cancer

\section{A Practical Method of Providing Pharmaceutical Care in the Intensive Care Unit: FASTHUG-MAIDENS Pharmacotherapy Best Practices Award, sponsored by Pfizer Canada}

Vincent H. Mabasa, Clinical Pharmacy Practice Leader, Burnaby Hospital, Burnaby, B.C., Canada

Douglas L. Malyuk, Clinical Pharmacy Specialist - Intensive Care, Royal Columbian Hospital, New Westminster, B.C., Canada

Elisa-Marie Weatherby, Clinical Pharmacist, Peach Arch Hospital, White Rock, B.C., Canada

Alice Chan, Clinical Pharmacist, University of Alberta Hospital, Edmonton, A.B., Canada

Background: Approaching patient work-ups using a formalized systematic process in the Intensive Care Unit (ICU) could potentially help pharmacists provide consistent and optimal pharmaceutical care. The mnemonic FASTHUG, developed for clinicians practicing in the ICU has been adapted to FASTHUG-MAIDENS so that pharmacists can utilize this tool to provide pharmaceutical care.

Objectives: To describe the FASTHUG-MAIDENS mnemonic and to quantify the percentage of drug-related problems (DRPs) that this tool can identify in intensive care patients.

Methods: The FASTHUG-MAIDENS mnemonic is an acronym for various drug related issues that the pharmacist considers when assessing the patient. Details are provided in the figure below. For ten work days, two clinical pharmacists tracked every patient encounter in the twentysix bed ICU. Data was collected on the DRPs identified for all patients assessed with and without the FASTHUG-MAIDENS mnemonic.

Results: Seventy-seven patients were included in this study. A total of 256 DRPs were identified with an average of 3 DRPs per patient encountered. There were 233 DRPs identified using the FASTHUGMAIDENS mnemonic which reflects a $91 \%$ capture rate.

Conclusions: The FASTHUG-MAIDENS mnemonic is a useful stepwise approach for pharmacists working in the ICU to identify the majority of drug related problems.

Key Words: Critical Care, Intensive Care, Pharmacy, Pharmaceutical Care

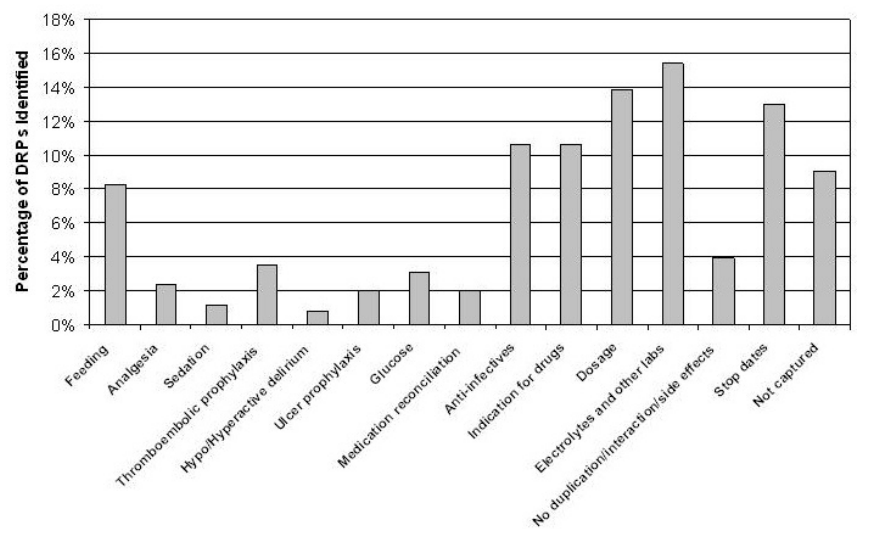




\section{Attitudes and Behaviors of Pharmacy Staff toward Near Misses: Reliability and Validity of a Survey Tool Safe Medication Practices Award, sponsored by Baxter Corporation}

Sarah Miller, Colette Raymond, Hannah Wheaton, Nick Honcharik Winnipeg Regional Health Authority, Winnipeg, Manitoba

Background: Near misses share may be early warning signals for errors. The purpose of this study was to develop and evaluate a survey to describe attitudes and behaviors of pharmacy staff toward near misses. Methods: The survey was developed through a literature review and field testing. Study subjects were pharmacists and technicians at a tertiary care hospital. Survey reliability was assessed with factor analysis and Cronbach's alpha and validated with focus groups and interviews. Qualitative description was used to identify themes.

Results: The survey response rate was $36 / 55$ (65.5\%). Eleven participants also attended interviews/focus groups. Although $67 \%$ of survey respondents had experienced a near miss within 3 months, only $10 \%$ reported the occurrence. Study subjects could identify pharmacy process changes that did or could result from near misses and identified learning from others' near misses. Some participants felt personally responsible for near misses, however many minimized the importance of near miss reporting in the context of a busy workday and onerous forms. An educational need about near misses and reporting within a non-punitive error reporting system was observed.

Conclusions: We describe a reliable and valid survey of pharmacy staff attitudes and behaviors toward near misses and reporting.

Key Words: Near misses, attitudes and behaviors

\section{Medication Reconciliation and the Role of the Pharmacy Technician: A Pilot Project with Acute Care Cardiac Services}

\section{Safe Medication Practices Award, sponsored by Hospira} Healthcare Corporation

Nadia Chishti, BSc Pharm candidate (3rd Year Student), Trudy Arbo (Clinical Pharmacy Practice Leader, NB Heart Centre), Moira Wilson (Director Pharmacy Services, Regional Health Authority B, Zone 2), Jaclyn LeBlanc (Clinical Practice Leader, Research and Education, Regional Health Authority B, Zone 2), Francine Bordage (Admin Director, NB Heart Centre), Alice Damon (Patient Safety Coordinator, Regional Health Authority B, Zone 2), Sandy Wilson

Study Question: Is the utilization of a pharmacy technician to perform the Best Possible Medication History (BPMH) for the medication reconciliation process as effective as usual practice?

Background: Up to $27 \%$ of prescribing errors in hospitals are caused by incomplete medication records at the time of admission, and 54\% of patients are reported to have at least one unintended discrepancy in their admission medication record. Medication reconciliation is labor intensive and time consuming for busy clinical staff. One solution is to utilize pharmacy technicians to perform the BPMH.

Methods: A retrospective chart review was performed on 200 randomly selected patient charts.

Results: The BPMHs taken by the pharmacy technician were consistently more legible and used at least two sources of information $94 \%$ of the time as compared to $6 \%$ with nursing staff. Postimplementation, there was a $16 \%$ increase in the identification of whether a patient had drug coverage or not, and a $5 \%$ increase of allergies noted. Overall, the implementation of a pharmacy technician for performing BPMHs showed an overall improvement in the quality of medication reconciliation by $9 \%$.
Conclusion: With the proper training, pharmacy technicians can alleviate the workload of other health care professionals with the medication reconciliation process and have demonstrated their ability to take an accurate and complete BPMH.

Key Words: Medication Reconciliation, Pharmacy Technician, BPMH

\section{Evaluation of Creatinine-Based Formulas in Dosing Adjustment of Cancer Drugs Other Than Carboplatin}

\section{Specialties in Pharmacy Practice Award, sponsored by Bristol-Myers Squibb Canada}

Sarah Jennings, ${ }^{1}$ Mário L. de Lemos, ${ }^{2}$ Adeera Levin, ${ }^{3}$ Nevin Murray ${ }^{2}$ ${ }^{2}$ At the time of the study, BC Cancer Agency; ${ }^{2} B C$ Cancer Agency; $B C$ Provincial Renal Agency, Vancouver, $B C$

Background: Glomerular filtration rate (GFR) can be calculated using Cockcroft-Gault (CG) or Modified Diet in Renal Diseases (MDRD) Study formulas. We previously showed them to be of similar accuracy for dosing carboplatin in gynecological cancer patients. We now compared them for other renally-excreted cancer drugs in the general cancer population.

Methods: Patient data were collected retrospectively at the BC Cancer Agency. Primary outcome was the proportion of patients receiving a different dose due to the GFR difference and dose adjustments from the patients' treatment protocols. Secondary outcome was GFR concordance using the Bland and Altman plot. Difference of $>30 \%$ was assumed to be clinically significant.

Results: 313 patients were evaluated (40\% male): median 56 y, 67.5 $\mathrm{kg}, 166 \mathrm{~cm}$, serum creatinine $74 \mathrm{micromol} / \mathrm{L}$. Median GFR was: CG formula, $86.8 \mathrm{~mL} / \mathrm{min}$ (mean $91, \mathrm{SD}_{ \pm} 30$ ) vs. MDRD formula, 87.6 $\mathrm{mL} / \mathrm{min}$ (mean $88, \mathrm{SD} \pm 26$ ). $8.6 \%$ of patients would receive a different dose. GFR concordance was found in $82.1 \%$ of patients. Conclusions: CG and MDRD formulas have good concordance, with $<10 \%$ of patients receiving a different dose. MDRD formula may be a reasonable alternative to the CG formula for dosing cancer drugs which require adjustments for renal dysfunction.

Key words: renal function, glomerular filtration rate, antineoplastic agents

Supported by a research grant of the Canadian Society of Hospital

Pharmacists

\section{Evaluation of Parenteral Vitamin B12 Administration in Hemodialysis Patients} Specialties in Pharmacy Practice Award, sponsored by Hospira Healthcare Corporation

Victoria Su' ${ }^{l}$; Karen Shalansky'; Jacek Jastrzebski ${ }^{2}$; Guiyun $\mathrm{Li}^{2}$; Alan Martyn ${ }^{3}$; Choi K Yeung'; Nadia Zalunardo

${ }^{1}$ Pharmaceutical Sciences CSU, ${ }^{2}$ Division of Nephrology, ${ }^{3}$ Department of Medicine, Vancouver General Hospital, University of British Columbia, Vancouver, Canada

Background: Methylmalonic acid (MMA) may be a better indicator of vitamin B12 deficiency in hemodialysis (HD) patients than serum B12 levels. We assessed vitamin B12 status and the effect of parenteral vitamin B12 on hematologic indices and MMA levels in macrocytic HD patients and a group of normocytic controls.

Methods: This was a 12 week prospective open-label cohort study of $62 \mathrm{HD}$ patients (34 macrocytic, 28 normocytic). Patients were on HD $\geq 3$ months, on stable doses of darbepoetin $\geq 6$ weeks and iron replete. 


\section{CSHP NATIONAL AWARDS PROGRAM / PROGRAMME NATIONAL DES PRIX 2010 DE LA SCPH}

Vitamin B12 $1000 \mathrm{mcg}$ IV was given once weekly post-HD for 4 weeks. Data was collected at baseline, weeks $+2,+6$ and +12 .

Results: At baseline, all patients had serum B12 levels $>200 \mathrm{pmol} / \mathrm{L}$ and there was no difference in serum B12 levels between groups (504 vs. $571 \mathrm{pmol} / \mathrm{L}, \mathrm{p}=0.18)$; MMA was higher in the macrocytic group ( 0.56 vs. $0.48 \mathrm{umol} / \mathrm{L}, \mathrm{p}=0.048$ ). Following IV vitamin $\mathrm{B} 12$, the macrocytic group had a greater reduction in MMA (-0.064 vs -0.0066 $\mathrm{umol} / \mathrm{L} / \mathrm{wk}, \mathrm{p}=0.004)$ which was sustained to week 12 . There was no improvement in $\mathrm{Hg}$, reticulocyte count, or MCV compared to baseline in either group. Median darbepoetin dose remained constant at $20 \mathrm{mcg} /$ week in both groups.

Conclusions: IV vitamin B12 led to a sustained decline in MMA levels in macrocytic patients suggesting possible functional vitamin B12 deficiency at baseline. However, there were no significant changes in $\mathrm{Hg}$ or darbepoetin dose. These preliminary findings do not support routine parenteral vitamin B12 supplementation in HD patients. Key Words: Anemia, Hemodialysis, Macrocytosis, Vitamin B12

\section{The Development and Evaluation of a Student Pharmacist Clinical Teaching Unit Utilizing Peer Assisted Learning}

Teaching, Learning, and Education Award, sponsored by Eli Lilly Canada

Adrienne Lindblad', Jason Howorko ${ }^{l}$, Richard Cashin ${ }^{l}$, Cornelius Ehlers', Carmen Leung', Erin Cashin', Cheryl Cox ${ }^{2}$

${ }^{1}$ Alberta Health Services, Red Deer, AB

${ }^{2}$ Faculty of Pharmacy and Pharmaceutical Sciences, University of Alberta, Edmonton, $A B$

Background: Requests for student pharmacist experiential placements are increasing. New models for hospital experiential education may increase capacity.

Objective: To create and assess a student pharmacist clinical teaching unit (CTU) that increases the preceptor to student ratio from 1:1 to 1:6.

Methods: Peer assisted learning, longer rotation durations and staggered rotation start dates, were used. Students cared for individually assigned patients and critically reviewed the work of their peers. Daily CTU rounds allowed students to present their patients to improve clinical reasoning skills. Program evaluation was accomplished through surveys of students and preceptors as well as comparison of workload statistics with the previous year.

Results: Five final year students participated in the CTU from January - April 2009. Preceptors reported no increase in workload with the CTU. More drug-related issues (DRI) were documented during the CTU than the previous year (768 and 151, respectively). Both students and preceptors were satisfied with the model and felt that the students' confidence, independence, judgment, time management skills and responsibility were increased with the CTU model compared to the traditional 1:1 preceptorship model.

Conclusion: The CTU increased training capacity while enhancing the educational experience for students and preceptors and increasing the number of DRI documented.

Key Words: education, capacity, peer assisted learning 